\title{
Der allgegenwärtige Schatten des Kommunismus im tschechischen Parteiensystem
}

\author{
Lukáš Novotný1,* \\ 1 Jan-Evangelista-Purkyně-Universität, Ústí nad Labem, Tschechische Republik \\ * E-Mail: novotny.l@centrum.cz
}

\section{Zusammenfassung}

In keinem anderen Land des ehemaligen Ostblocks finden wir eine kommunistische Partei, die gesellschaftlich ähnlich bedeutend ist wie die Kommunistische Partei Böhmens und Mährens (KSČM) in der Tschechischen Republik. Ihre Erfolge und die Tatsache, dass die Partei sich von ihrem Wirken in der Zeit des orthodoxen Kommunismus nie „losgesagt“ hat, sondern darin bis heute eine Quelle der Inspiration für ihre Tätigkeit sieht, macht sie zu einer politischen Anomalie ersten Ranges im Rahmen der gesamten Europäischen Union. Ziel dieser Studie ist es, die grundlegenden Prädispositionen zu definieren, die hinter der Tatsache stehen, dass genau diese Partei, die nach 1989 als antisystemischer Totengräber eines undemokratischen Regimes galt und mit der für die Zukunft niemand mehr wirklich rechnete, bis heute zu den stabilsten Elementen in der tschechischen Politik gehört.

\section{Schlüsselwörter}

Kommunistische Partei, KSČM, Kommunismus, Tschechien, Parteiensystem, Vergangenheitsbewältigung

\section{The omnipresent shadow of communism in the Czech party system}

\begin{abstract}
In no other country of the former Eastern Bloc, we can find a Communist Party that is similarly strong and socially anchored, as is the case of the Communist Party of Bohemia and Moravia (KSČM). Its successes and the fact that the Party has not been able to „cut off" itself from its past and from Orthodox communism yet, but still sees it as the source and inspiration for its political activity, make it a political anomaly throughout the European Union. The aim of this study is to define the basic predisposition for the success of the party and the fact that it is precisely this party that before i 989 consistently suppressed everything that would strengthen democracy. No one really expected this party to have a future, yet it still represents one of the most stable elements of Czech politics.
\end{abstract}

\section{Key words}

Communist Party, KSČM, communism, Czech Republic, party system, dealing with the past

The author has declared that no competing interests exist. 


\section{Einleitung}

In keinem anderen Land des ehemaligen Ostblocks finden wir eine kommunistische Partei, die gesellschaftlich ähnlich bedeutend ist wie die Kommunistische Partei Böhmens und Mährens (KSČM) in der Tschechischen Republik. Ihre Erfolge und die Tatsache, dass die Partei sich von ihrem Wirken in der Zeit des orthodoxen Kommunismus nie "losgesagt" hat, sondern darin bis heute eine Quelle der Inspiration für ihre Tätigkeit sieht, macht sie zu einer politischen Anomalie ersten Ranges im Rahmen der gesamten Europäischen Union (Grzymala-Busse 2002). In keinem anderen Land spielt eine aus einer kommunistischen Partei hervorgegangene und nur minimal transformierte Partei eine ähnliche Rolle im Parteiensystem, in keinem anderen Land hat sie eine so große und zugleich stabile Unterstützung durch die Wählerschaft (Hanley 2002). Sinn dieser Studie soll es sein, die grundlegenden Prädispositionen zu definieren, die hinter der Tatsache stehen, dass genau diese Partei, die nach 1989 als antisystemischer Totengräber eines undemokratischen Regimes galt und mit der für die Zukunft niemand mehr wirklich rechnete, bis heute zu den stabilsten Elementen in der tschechischen Politik gehört. Der Studie liegen vorhandene Untersuchungen zur Problematik der KSČM sowie relevante Meinungsumfragen zugrunde. Die Hauptaspekte, denen hier besondere Aufmerksamkeit gewidmet werden soll, sind: Abstimmung der Interessen zwischen Hardlinern und Pragmatikern, das Bemühen der Parteieliten um
Wieder-Legitimierung der KSČM, die stabile Wählerbasis, der "Klassencharakter" und der Einfluss des historischen Gedächtnisses.

Die Frage, ob die KSČM verboten werden sollte, war nach dem November 1989 Gegenstand zahlreicher Überlegungen und Initiativen. Zum Verbot kam es allerdings nicht. Kritisch äußert sich hierzu in seinen Erinnerungen auch der damalige tschechische Premierminister Petr Pithart, ein direkter Akteur der Entwicklung nach 1989. In seiner Arbeit „Das Jahr '89" von 2009 schreibt er: "In den letzten Jahren wird ständig wiederholt: Damals ist ein Fehler passiert, damals hätten die Kommunisten verboten werden müssen, aber wenn das damals nicht gemacht wurde, heute ist es zu spät dafür" (Pithart 2009, I22f.). Dabei war es neben Václav Havel gerade auch Petr Pithart, der immer wieder betont hatte, die kommunistische Partei werde sich bald in Misskredit gebracht haben und von selbst aufhören, eine relevante politische Kraft im Land zu sein (Havel 2006, 49).

Diese Hoffnung hat sich nicht erfüllt. Die KSČM hatte in den Parlamentswahlen 2013 mit ihrem Programm 74I Tsd. Bürgerinnen und Bürger angesprochen, das waren im Endergebnis $14,9 \%$ der abgegebenen Stimmen (siehe Säulendiagramm). Die Partei hatte nahezu I50 Tsd. Wählerstimmen mehr erhalten als im Jahr 20IO. Ähnlich war der Aufstieg der KSČM auch in den Wahlen in den Regionen. Während die Partei 2008 nicht ganz 440 Tsd. Stimmen (ca. $15 \%$ )

Abbildung 1: Stimmenanteile der Kommunisten in den Parlamentswahlen (Angaben in \%)

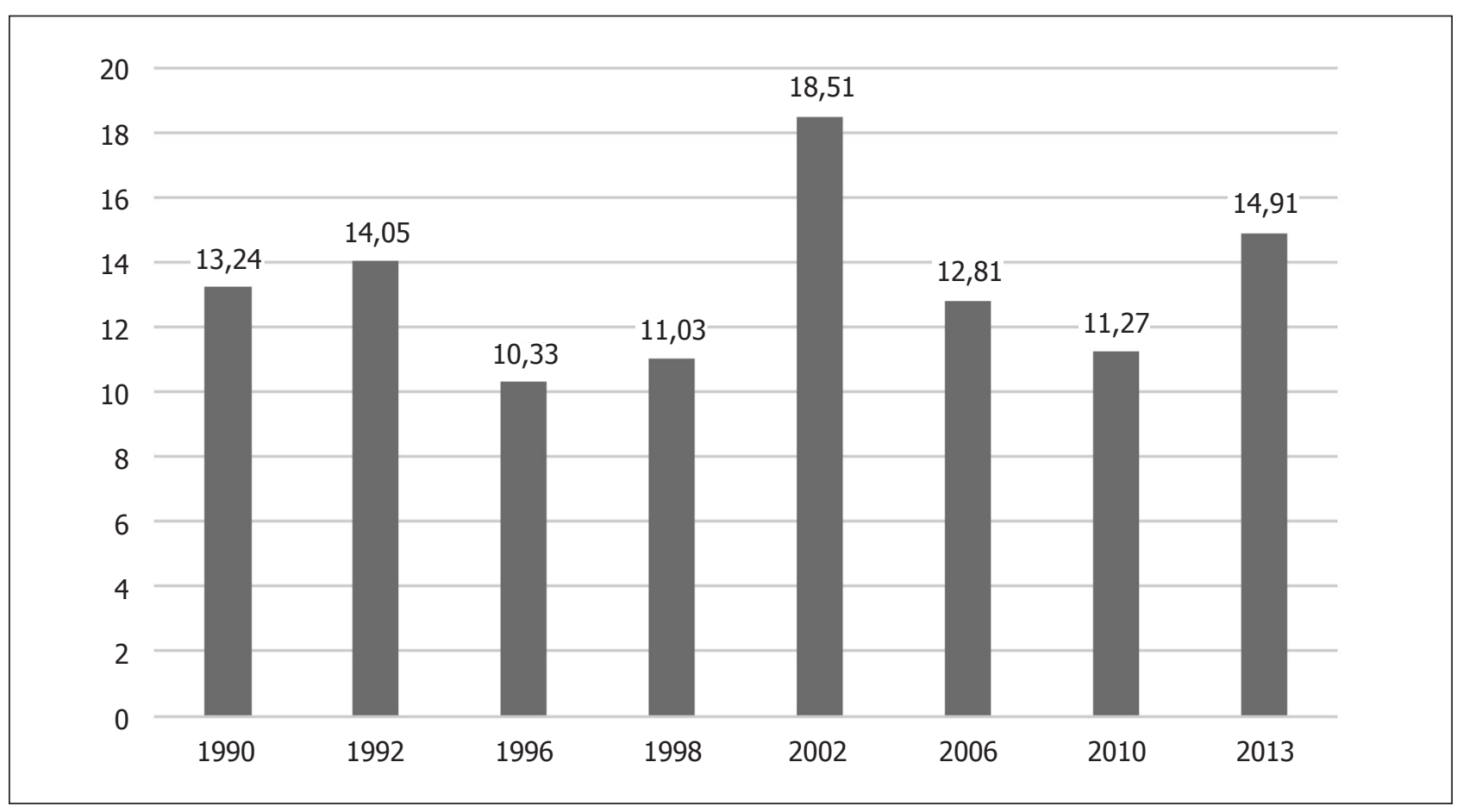

Quelle: www.volby.cz 
erhalten hatte, waren es 2012 rund Ioo Tsd. Stimmen mehr, d. h. mehr als $20 \%$ aller abgegebenen Stimmen. Die letzten Parlaments- und Kommunalwahlen haben also bestätigt, dass die KSČM ein konstanter Bestandteil des tschechischen Parteiensystems ist. Sie ist darüber hinaus eine der zwei etablierten Linksparteien und heute inzwischen (zusammen mit der Sozialdemokratischen Partei ČSSD und der Demokratischen Bürgerpartei ODS) eine von nur drei Parteien, die seit 1992 ohne Unterbrechung im tschechischen Parlament vertreten sind (Vodička 2005). Von diesen drei ist sie die Partei mit der stabilsten Basis.

Die KSČM ist voll in das tschechische politische System integriert, nimmt an Parlaments-, regionalen und kommunalen Wahlen teil und hat ihre Vertreter in den kommunalen und regionalen Vertretungsorganen und im Parlament. Bedingt durch die Haltung der Sozialdemokraten (ČSSD), die es bislang ablehnen, auf Regierungsebene eine Koalition mit Kommunisten einzugehen, besteht zwar für die KSČM keine Hoffnung, einen Anteil an der politischen Macht in Tschechien zu erlangen, allerdings gelingt es ihr mit ihrer Protesthaltung spezifische entfremdete subkulturelle Gruppen mit spezifischen politischen Verhaltensmustern, die wünschen, dass ihre Stimme in der Öffentlichkeit gehört wird, mit Erfolg zu organisieren und zu mobilisieren (Waller 1995). Sie vertritt die kompromisslose Opposition, die die Proteststimmen sammelt (Thieme 2007).

\section{Hardliner und Pragmatiker}

Wie immer wir die frühe oder spätere Geschichte der kommunistischen Partei beurteilen, Tatsache ist, dass sie bereits seit ihrer Gründung die politischen Hoffnungen eines ganz bedeutenden Segments der tschechischen Gesellschaft repräsentiert hat. Wir müssen zur Kenntnis nehmen, dass es sich um eine politische Partei handelt, die kontinuierlich seit mehr als 90 Jahren im tschechischen politischen System verankert ist und für die ein starker organisatorischer Background und spürbare politische Legitimität kennzeichnend sind (Thieme 2007). Auch nach 1989 konnte die KSČM ihre starke Position in der tschechischen Politik halten, obwohl sie in der Gesellschaft als markant antisystemisches politisches Subjekt wahrgenommen wurde. Die Probleme, die im Zusammenhang mit der Transformierung von Gesellschaft und Wirtschaft faktisch seit den Neunzigern und auch in den nachfolgenden Jahren zu Tage traten, machten es den Kommunisten möglich, für einen Teil der Wähler eine reale Wahl-Alternative zu bleiben und bis zu einem gewissen Grad ihren Einfluss sogar auszubauen, ohne dass sie dafür zu grundlegenderen innerparteilichen Reformen gezwungen gewesen wären. Gemessen an den Wahlergebnissen scheint der auf dem
Parteikongress 1993 eingeschlagene Weg, also zu einer Zeit, da die Befürworter der Beibehaltung der bisherigen politischen Linie definitiv überwogen, politisch produktiv gewesen zu sein (Grzymala-Busse 2002, $22 \mathrm{ff}$.). Der führende marxistisch-leninistische Flügel mit Miroslav Grebeníček an der Spitze konnte seine Vorherrschaft in der Partei auch in den nachfolgenden Jahren behaupten. Damals war es ein Machtkampf zwischen Reformisten und eben jenen Verfechtern der alten Ordnung. Was die Parlamentswahlen betrifft, scheint der Sieg des konservativen Flügels für die Zukunft der Partei der richtige Weg und der bewusste konzeptionelle Schritt zur Positionierung im Parteiensystem Tschechiens zu sein (Mannewitz 2012; Maškarinec 20I7). Doch die Regionalwahlen 2016 waren für die Partei unzufriedenstellend und ihre Ideologen müssen offensichtlich die Parteistrategie neu überlegen. Dabei werden die Regionalwahlen als Test vor der Wahl zum Abgeordnetenhaus verstanden. Diese finden in diesem Jahr statt.

Auch sonst waren die Trends in der Partei zu Beginn der Neunzigerjahre eindeutig: Die Parteieliten lehnten eine innerparteiliche Reform ab, und letztendlich wünschten auch die Wähler mehrheitlich keine Veränderungen. Das waren jene, die traditionell die Kommunistische Partei der Tschechoslowakei (KPTsch) gewählt hatten und die in einer Zeit der Transformation, der Auseinandersetzungen mit der Vergangenheit des jüngst zu Ende gegangenen totalitären Regimes und des Drucks antikommunistischer gesellschaftlicher Kräfte in ebendieser KPTsch ein gewisses stabilisierendes Element sahen. Rechnen wir nun noch die langjährige starke Verankerung der KPTsch im tschechoslowakischen Parteiensystem hinzu, gefestigt durch die vierzigjährige kommunistische Vorherrschaft, aber auch die allgemeine Schwäche und fehlende einheitliche Strategie der Parteiführung und das Nichtvorhandensein durchdachter Schritte der Reformwilligen, dann erkennen wir, dass die Zeit für die Umwandlung der KPTsch in eine moderne Partei postkommunistischen Zuschnitts, die sich auch auf verbaler Ebene von dem Begriff „kommunistisch" in ihrem Namen verabschiedet, nicht besonders günstig war. Genau hier erkennen wir die Ansätze für das spätere Agieren der KSČM als Nachfolgepartei der KPTsch nach dem Zerfall der tschechoslowakischen Föderation im Jahr 1992 (Vodička 20IO).

Hinzugefügt werden muss, dass der Partei bis heute diese gewisse organische und ideologische Zerrissenheit eigen ist. Zwar haben sich die innerparteilichen Plattformen aufgelöst, aber in der Partei gibt es weiterhin liberale Progressisten, die den Weg einer modernen radikalen Linkspartei, vor allem nach dem Vorbild der deutschen Die Linke gehen möchten, wie auch orthodoxe Marxisten, die vernarrt in die Vergangenheit sind und Reformen ablehnen (Backes/Moreau 2008, 555). Zwischen beiden Positionen stehen die Parteiführer, 
ausgleichend und die eine oder die andere Gruppe zur Mäßigung bewegend, wodurch es ihnen gelingt, einen gewissen Status quo aufrechtzuerhalten (GrzymalaBusse 2002, 89ff.). Dieser Zwiespalt, der sich u. a. in nicht eben ungetrübten Beziehungen zwischen Parteiführung, Mitgliederbasis und Wählern bzw. Sympathisanten äußert, ist aus der Perspektive der Debatten um den antisystemischen Charakter der KSČM interessant. Während die Strömung, die die Modernisierung der Partei anstrebte, als relevante Kraft inzwischen kaum noch vorhanden ist, haben die "Zentristen“ aus der Parteielite, für die ihr Engagement in der KSČM auch ein guter Job mit der Aussicht auf eine lange politische Karriere als "ewig“ oppositionelle Abgeordnete und andere politische Vertreter ist, die Macht in der Partei übernommen. Auf der Ebene der politischen Eliten beobachten wir also eine gewisse Variante der Two-Level Game-Theorie von R. D. Putnam (I988), wobei KSČMMitglieder und -wähler die einheimische Ebene der politischen antisystemischen Agenda darstellen und die Parteiführung, die sich im Allgemeinen innerhalb des demokratischen Spektrums bewegt, die äußere Ebene. Tatsache ist, dass die Führung der KSČM einem nicht nachlassenden Druck der starken radikal konservativen Strömung standhalten muss, einer Strömung, die paradoxerweise auf der Leitungsebene weit weniger stark vertreten ist. Darin besteht wahrscheinlich jener Pragmatismus der Parteiführung und wir können davon ausgehen, dass zumindest mittelfristig die pragmatische Denkweise die Oberhand behalten wird (Mannewitz 2OI2, 3II).

\section{Der Weg zur Wieder-Legitimierung der KSČM}

Die spezifische ideologische Position der Partei lässt sich in mehrere Entwicklungsphasen aufschlüsseln. Charakteristisch für den Anfang der Neunzigerjahre ist eine gewisse Blockierung der Transformation der kommunistischen Partei. Wir sprechen von der „Strategie des Rückzugs des linken Flügels“ (Ishiyama/Bozóki 200I, 34) und der Vorherrschaft des orthodox-kommunistischen Kurses Anfang der Neunzigerjahre, während der Parteimitglieder, die für eine Modernisierung waren, aus der Partei ausschieden. Danach folgt die Etappe der Stabilisierung und des Wachstums (1993-2004), die bis zur Position eines gefragten politischen Partners etwa ab Mitte des vorigen Jahrzehnts führte (Novotný 2oII, 406). Im Jahr 2002 erzielte die Partei ihr bisher bestes Ergebnis. Bezeichnend für die letzten Jahre sind die Bestrebungen, die KSČM in die politische Verantwortung einzubeziehen. Dies geschieht vor allem auf regionaler Ebene, wo die KSČM einen von I3 Hauptleuten stellt und Koalitionspartner in mehreren regionalen Regierungen ist. Das führt zu einer gewissen Wieder-Legitimierung der Partei. Sie ist auf dem besten Weg, ihre früher fast totale politische Isolation auch auf oberster politischer Ebene zu durchbrechen, und steuert auf eine vollständige Kooptation in das politische System Tschechiens zu. Dauerhafte Unterstützung schöpft die KSČM bis zu einem gewissen Grad aus ihrer Position als einziger wirklicher Herausforderer des Nach-Wende-Establishments, wobei gerade der langjährige Ausschluss der Kommunisten aus dem System es erst möglich gemacht hat, dass sie sich als Einzige einer attraktiven ProtestRhetorik bedienen können. Die Kommunisten empfinden das allerdings als Unrecht und wenden ein, sie seien die einzige politische Kraft, die keines der Probleme der gesellschaftlichen und wirtschaftlichen Transformation nach 1989 verschuldet habe. Die Richtung, in die die Bemühungen der KSČM gehen, ist eindeutig: weg von der Position einer Anti-System-Partei und hin zur systemloyalen Partei (Novotný 2004).

\section{Stabile Wählerschaft}

Bezeichnend für Wähler der KSČM ist die Beständigkeit ihres Wahlverhaltens (Ishiyama 1999). Etwa zwei Drittel von ihnen haben in der Vergangenheit nie eine andere Partei gewählt. In den Jahren 1990-2013 waren es rund 400 Tsd. Menschen, die die kommunistische Partei kontinuierlich unterstützt haben. Die KSČM kann sich also auf mehrere Hunderttausend Stimmen traditioneller Wähler stützen und sie profitiert von einer außerordentlichen Wahldisziplin und Loyalität (Novotný 2004). Daneben gewinnt die KSČM kontinuierlich weitere Unterstützung außerhalb ihres eigenen ideologischen Umfelds. Diese Tatsache widerspricht der seit langem von Politikern und Politologen immer wieder geäußerten Auffassung, es gebe eine Tendenz zur „biologischen Lösung der kommunistischen Frage". Das immer höhere Durchschnittsalter der KSČM-Wähler ist zwar empirisch nachweisbar, seine Dringlichkeit und vor allem seine Dynamik werden aber durch die Sympathien latenter Wähler gebremst. Betrachtet man die Ergebnisse der KSČM, dann wird deutlich, dass das Altern ihrer Sympathisanten bislang keine spürbaren Folgen für die Partei hatte. Es gelingt ihr, mit ihren programmatischen Prioritäten neue Wählergruppen anzusprechen, in erster Linie unzufriedene Sozialdemokraten und auch Nichtwähler. Ein Problem für die Partei ist das „Schmelzen" des sog. harten Kerns ihrer Wähler, dazu kommen das Altern und die Sterberate der Mitgliederbasis. Die Partei hat derzeit 5I ooo Mitglieder. Das Durchschnittsalter der KSČM-Wähler ist zwar immer noch deutlich niedriger als das der Parteimitglieder, es nimmt aber nachweislich seit den Neunzigerjahren zu. Während es im ersten Jahrzehnt nach der Wende zwischen 53 und 54 Jahren oszillierte, liegt es gegenwärtig bei annähernd 60 
Jahren (zum Vergleich: Das Durchschnittsalter der Wähler der übrigen Parteien beträgt etwa 44 bis 45 Jahre) (Kunštát 2OI3).

Analog zum Altern der Wählerschaft altert auch die Mitgliederbasis der KSČM, deren Zahl permanent abnimmt. Das Durchschnittsalter der KSČM-Mitglieder beträgt derzeit annähernd 73-74 Jahre, während es 1996 nicht ganz 62 Jahre betrug. Bei der Gründung der KSČM im März 1990 gab die Partei ihre Mitgliederzahl mit über 700 Tsd. an. Diese Zahl war allerdings überzogen. Trotz der Massenaustritte Anfang der Neunzigerjahre hatte die KSČM im Jahr 1992 aber immer noch 355 Tsd. Mitglieder, das war rund ein Drittel der tschechischen Mitgliederbasis der KPTsch in den letzten Jahren vor der Wende. Die Mitgliederbasis der tschechischen kommunistischen Partei war zu dieser Zeit viel stärker als die anderer (post-)kommunistischer Nachfolge-Parteien, und sie war auch größer im Vergleich zu den anderen tschechischen politischen Parteien (Grzymala-Busse 2002, 77).

Die Geschwindigkeit der natürlichen Schrumpfung der KSČM-Mitgliederbasis hat sich in den letzten Jahren bei etwa $8 \%$ jährlich eingependelt (d. h. rund 5 Tsd. Mitglieder weniger pro Jahr). Die starke natürliche Abnahme der Mitgliederzahl bedeutet aber auch den Wechsel von der Massenpartei zur Wahlpartei - in Abhängigkeit davon, wie sich die Korrelation zwischen Mitgliederzahl und Wahlergebnissen allmählich verringert. Beunruhigend für die KSČM dürfte nicht nur die absolute Schrumpfung der Mitgliederbasis sein, sondern auch deren Altersstruktur. Mehr als die Hälfte der Mitglieder ist älter als 70 Jahre. Trotzdem bleibt die KSČM auch weiter die zahlenstärkste Massenpartei in der Tschechischen Republik. Keine andere politische Partei hat in ihrer Wählerschaft einen vergleichbar hohen Anteil ehemaliger Mitglieder des einstigen Parteihegemons. Andererseits sind viele, die vor ' 89 Kommunisten waren, der "Sache des Sozialismus" nicht treu geblieben und sind heute Mitglieder anderer Parteien quer durch das Parteienspektrum. Nur zwei Fünftel der gegenwärtigen KSČM-Wähler besaßen vor 1989 den Parteiausweis der Kommunistischen Partei der Tschechoslowakei (Kunštát 2013, 243). Die These, die KSČM werde größtenteils oder überwiegend von „ehemaligen Kommunisten“ gewählt, ist nicht absolut gültig. Die Mehrzahl der heutigen Wähler dieser Partei hatte mit der KPTsch vor 1989 nichts gemein. Das bedeutet, die Partei findet politische (Wähler-)Unterstützung auch außerhalb ihres traditionellen kommunistischen Umfelds (Novotný 2004, 2IO). Die KSČM hat sich ihre personelle Kontinuität mit der KPTsch wesentlich stärker auf interner Ebene, der Ebene der Parteistrukturen bewahrt, als auf der Linie ihrer Wählerschaft.

\section{„Klassencharakter" der KSČM}

Die Tätigkeit der KSČM ist vollständig kompatibel mit der Entwicklung der Hauptkonfliktlinie in der tschechischen Politik, der sozioökonomischen Konfliktlinie, die sich Mitte der Neunzigerjahre konsolidierte und die auch entscheidend für die Strukturierung des tschechischen Parteiensystems ist (Vodička 20IO, I67). Die tschechische Linke stabilisierte sich dabei in Form zweier maßgeblicher Parteien, der von 1948 bis I989verbotenen und im Jahr 1990 wiedergegründeten Tschechischen Sozialdemokratischen Partei und der nicht reformierten KSČM, die manche Politologen - mehr oder weniger zu Recht - für eine Anti-System-Partei halten (Kubát 2oIO, 92). Eine weitere starke Konfliktlinie - zumindest auf der Ebene des Wählerlagers und bis zu einem gewissen Grad auch im Rahmen der Mitgliederbasis - lässt sich in den Ansichten über die Rolle des Staates im sozialen und wirtschaftlichen Bereich verfolgen. In die konkrete Parteiidentität linksgerichteter Wähler treten nämlich unabhängig und mit unterschiedlicher Intensität weitere, untereinander integral vernetzte Faktoren, die für die Spaltung des linken Wählerlagers (trivial gesagt in „kommunistische“ und „nicht kommunistische“ Wähler) wichtiger sind:

I) die Dimension der „Werte“-Unterstützung oder, im Gegensatz dazu, der Entfremdung von der demokratischen Regierungsform und den Ansichten über die politische und wirtschaftliche Entwicklung nach 1989

2) die Dimension des Verhältnisses zur Vergangenheit vor 1989, bei kommunistischen Wählern gibt es eine positive emotionelle Bindung an diese Ära

3) die Dimension der außenpolitischen Orientierung („Ost“"-West"-Konfliktlinie).

Betrachten wir die "historische“ KPTsch, so stellen wir fest, dass sie hinsichtlich der sozialen Basis bereits seit ihrer Gründung auf die Arbeiterklasse bzw. allgemein auf die unteren sozialen Schichten orientiert ist, die durchgängig (bis zum Jahr 1989) die überwiegende Mehrheit der Mitglieder bildeten (Novotný 2010, 406). Diese geschichtliche Rolle einer "Vorhut" bzw. politischen Repräsentantin der Arbeiterklasse erfüllt die KSČM bis zu einem gewissen Grad auch heute, obwohl es symptomatisch für sie ist, dass es ihr nur begrenzt gelingt, die „aktive" Arbeiterschaft anzusprechen (die in Parlamentswahlen eher sozialdemokratisch wählt). Trotzdem ist es der KSČM gelungen, einen relativ eigenständigen Platz in der Konfliktlinie "soziale Schichten" der tschechischen Politik einzunehmen. Gerade die feste Verankerung der Wähler-Klientel der KSČM in der Struktur der sozialen Spaltungen bietet eine Erklärung für die erhebliche Wählergunst und auch dafür, dass die kommunistische Partei vorwiegend für die Arbeiterklasse 
und die untere Mittelschicht attraktiv wurde (Mateju u. Kreidl 200I). Die Wählerschaft der KSČM besteht längst nicht mehr nur aus "Menschen des alten Regimes“, sondern sie repräsentiert bereits seit Beginn der Neunzigerjahre die realen Interessen bestimmter Segmente der Gesellschaft - und zwar Interessen, die primär sozial, jedoch nicht ideologisch bedingt sind. Der Hintergrund der Affinität eines beachtlichen Teils der Wähler zur KSČM ist also nicht notwendig die Wahrnehmung der Werte (Ideologie) des kommunistischen Regimes und der demokratischen Transformation, sondern es ist eine auf individueller Ebene gebildete sozioökonomische Bewertung („Interessen“) von Vergangenheit und Gegenwart (Handl 20IO). Für die Interpretation der Unterstützung der KSČM hat diese sozioökonomische Dimension Schlüsselfunktion. Die KSČM verkörpert die Tradition des Vergangenen, des „Vorteilhafteren“ und vertritt adäquat jene Interessengruppen, die von den Mustern des alten Systems, dessen Institutionen und bezeichnenderweise dessen etatistischer Sozialstruktur bestimmt werden und die mit einer gewissen Nostalgie auf die "gute alte sozialistische Zeit", die Zeit der Gleichheit und der „risikolosen Gesellschaft“ zurückblicken. Diese "sozialistische Mentalität" ist in den Gruppen stark verwurzelt, die mit wirtschaftlichen Schwierigkeiten zu kämpfen haben und die nicht in der Lage sind, neue und mit dem Milieu des "demokratischen Kapitalismus“" kompatible Lebensstrategien zu entwickeln. Die Folgen der radikalen Veränderungen im Gesellschaftssystem haben die natürliche soziale Basis der KSČM hervorgebracht, die nun ihrerseits die Erhaltung des alten Systems bzw. von dessen Teilen (vor allem der staatlichen gelenkten Wirtschaft) verteidigt. Anhänger der kommunistischen Partei sind hauptsächlich Menschen aus Gruppen mit niedrigen Einkünften (Novotný 20IO, 399). Nicht zufällig kommen sie sehr zahlreich aus Gruppen mit Einkünften auf dem Niveau der Altersrenten, denn der Kern der KSČM-Anhänger sind Menschen, die wirtschaftlich nicht aktiv sind, in der überwiegenden Zahl eben Altersrentner. Das Verhältnis von wirtschaftlich inaktiven und aktiven Wählern beträgt 2:I, wobei in der Gruppe der wirtschaftlichen aktiven Wähler manuell tätige Menschen (Arbeiter, Bauern und Landarbeiter, Beschäftigte mit geringer oder ohne berufliche Qualifikation) stark überwiegen. Zugleich besteht die Wählerschaft der KSČM zu zwei Dritteln aus Menschen über 60 Jahren und mit durchschnittlich niedrigerem Bildungsgrad. Drei Viertel der Wähler dieser Partei haben als höchste Bildungsstufe einen Berufsschulabschluss (ohne Fachabitur) erlangt. Wir dürfen daher die Wählerschaft der KSČM als das Wähler-Segment mit dem niedrigsten Bildungsstand in der Tschechischen Republik betrachten.

Generell handelt es sich also um den Teil der Bevölkerung, der permanent wirtschaftliche Unsicherheit verspürt und dessen Lebenschancen (subjektiv wie ob- jektiv) fatal vom Maß der Umverteilung öffentlicher Mittel und vom Engagement des Staates in der Sozialpolitik abhängig sind (Minkerberg 200o). Die soziale Zusammensetzung der kommunistischen Wählerschaft mit ihrem dominierenden Anteil wirtschaftlich nicht aktiver Menschen reicht jedoch als Indikator nicht ganz aus. Das Klassenwesen der KSČM wird erst deutlich, wenn wir uns ansehen, aus welchen sozialen Schichten der überwiegende Teil der heutigen Wähler im Rentenalter stammt. Das ergibt ein Bild, das den vielleicht höchsten Aussagewert hat: Abgesehen vom aktuellen sozialen Status gehörte die Hälfte der KSČM-Wähler während ihrer wirtschaftlich aktiven Zeit vorwiegend der Berufsgruppe der Arbeiter an. Über ein Drittel waren niedere Angestellte und technisches Personal (Verwaltungspersonal, Hilfsfacharbeiter) und nur etwa ein Zehntel der KSČMWähler können als gesellschaftliche Elite der Vorwendezeit bezeichnet werden (höhere Angestellte, Lehrer, leitende Angestellte, Angehörige der Sicherheitskräfte usw.). Ein ähnliches Schema, in dem Arbeiterberufe und niedere Angestelltenpositionen überwiegen, ist auch für das nähere soziale Umfeld kennzeichnend, also für Eltern, Lebenspartner usw. (Kunštát 2OI3, 289).

Der angenommene „durchschnittliche“ KSČMWähler hat einen Beruf erlernt, ist männlich, wohnt auf dem Dorf oder in einer Kleinstadt bis max. mittelgroßen Stadt, ist über 60 Jahre alt, Rentner (bzw. wirtschaftlich nicht aktiv), war in der Vergangenheit ein gewöhnlicher Beschäftigter, kleiner Angestellter in der Verwaltung oder Facharbeiter, der seine produktivste Lebenszeit in den Jahren der Normalisierung (in der Geschichte der Tschechoslowakei die Zeit nach 1968) hatte. Er selbst fühlt sich der Arbeiterklasse oder der unteren Mittelschicht zugehörig. Er stammt aus dem Arbeitermilieu, der manuell arbeitende Vater hat (hatte) einen Beruf erlernt, auch die Mutter hat (hatte) eine Berufsausbildung und arbeitete als einfache Angestellte in der Verwaltung oder war Arbeiterin (Stegmaier u. Vlachová 2008, S. 805808). Mit anderen Worten, nicht der Offizier der ehemaligen Volksarmee, auch nicht das ehemalige Mitglied des Parteiapparats sind die typischen KSČM-Wähler, sondern viel eher der einfache Industriearbeiter oder der Traktorist einer Landwirtschaftlichen Genossenschaft, nicht die Lehrerin oder die Leiterin der Propagandaabteilung beim Nationalausschuss, sondern viel eher die Hilfsköchin aus der Schulküche oder eine einfache Verwaltungskraft. Das Wählermilieu der KSČM hat deutliche Klassenwurzeln, im Rahmen der sozialen Schichtung ist es primär in der Arbeiterschaft und bei kleinen Angestellten (bzw. allgemein in den unteren Schichten) etabliert. Diese Indizien relativieren die verbreitete, oft mit einer gewissen Ironie geäußerte Ansicht, die kommunistische Partei könne gar nicht als die politische Sprecherin der arbeitenden Klasse gelten, für die sie sich ausgibt. Wir sehen aber, dass die KSČM legitim als die 
Verteidigerin der sozialen Interessen der „Menschen der Arbeit" auftritt, also als Partei mit traditionell linksorientiertem Esprit.

\section{Historisches Gedächtnis}

Die Position der KSČM kann natürlich nicht einseitig nur aus ihrem Klassenzusammenhang hergeleitet werden. Die KSČM hat sich - trotz Teilreformen - ihre „Authentizität" erhalten (Grzymala-Busse 1998). Diese ergibt sich aus dem spezifischen, historisch konturierten Gedächtnis (besonders aus der „Erinnerung an die Normalisierung “) des sog. harten Kerns der Parteimitglieder und Wähler, das so etwas wie eine Kontradiktion zur offiziell deklarierten Nach-Wende-Erinnerung an den Kommunismus ist. Die Wahlaffiliation zur KSČM knüpft sich an ein bestimmtes typisches individuelles und kollektives historisches Bewusstsein, das das Wurzelgeflecht des integralen kulturpolitischen Milieus ist und das die Verbindung zu den verlorenen "guten Zeiten" herstellt (Handl 20IO). Die positive Bewertung des kommunistischen Regimes, eine relativ starke politische (institutionelle) Entfremdung und geringe Legitimität der demokratischen Herrschaftsform in den Augen derer, die sich mit der KSČM identifizieren, sind stark beeinflusst vom Alter bzw. der Zeit der ersten politischen Sozialisierung. Wie wir bereits festgestellt haben, erhält die Partei die stärkste Wahlunterstützung von über Sechzigjährigen (Novotný 2010, 407).

KSČM-Anhänger sind in ihrer Mehrzahl „einfache Menschen" mit gewöhnlichen Schicksalen, deren produktive Jahre größtenteils in die Siebziger- und Achtzigerjahre fallen. Speziell die Reflektion der Jahre der Normalisierung ist bei ihnen eine Art gemeinsame Erfahrung ihrer Generation, für die die damalige Politik eher den Unterbau und aus psychosozialer Sicht eher den äußeren Rahmen darstellte. Verschiedenen soziologischen Sonden zufolge sind bestimmte eigenständige „Erinnerungsmuster" typisch, die eine enge Beziehung zwischen dem historischen Bewusstsein und den historischen Bildern und Stereotypen der kommunistischen Vergangenheit belegen, also zwischen einer spezifischen Gedächtnisstruktur und deren konkreten Vergegenwärtigung in der "kommunistischen“ politischen Identität (Oschlies 2000, 34). Im Gegensatz zum typischen liberaldemokratischen Diskurs, der den Stillstand, die Unterdrückung, das Unrecht, den gesellschaftlichen Verfall und eine alles durchdringende Resignation für die Zeit vor 1989 proklamiert und immer wieder hervorhebt, exponieren sie alles "Gute" der Vorwendezeit: Aufbau, Aufschwung, Ruhe, zufriedenes Leben, Ordnung, Stabilität, Gerechtigkeit, Sieg der „wahren“ Idee (Moreau 20IO, 378). Die Kraftlinien dieser rückwärts, in die Vergangenheit gewandten Gegenidentität überschneiden sich in der Gegenwart mit der Erfahrung der (maroden, uneffektiven, korrumpierten, sozial nicht intakten) kapitalistischen Demokratie (Novotný 2007).

Zwei Drittel der KSČM-Wahler wünschen die Wiederherstellung des kommunistischen Systems, aber nur ein Fünftel meint, die Rückkehr zur alten Gesellschaftsordnung sei möglich. Der relativ geringe Anteil derjenigen, die chiliastisch an der Vorstellung einer baldigen Rückkehr der kommunistischen Herrschaft festhalten, zeugt $u$. a. auch davon, dass nur noch eine kleine Minderheit der Wähler dieser Partei die Hoffnung auf eine authentische Restauration des "Sozialismus“ in sich nährt, (dagegen halten sie zwei Fünftel der Wählerschaft für völlig ausgeschlossen). Auch der überwiegende Teil derer, die die Rückkehr zum Kommunismus begrüßen würden, hat diesen Wunschtraum aufgegeben, ebenso die Parteimitglieder, die, wie es scheint, den Glauben an eine revolutionäre Zukunft verloren haben - drei Viertel von ihnen halten die Rückkehr zum Kommunismus nicht für wahrscheinlich oder möglich (Kunštát 2013, 274f.).

Die Wähler der KSČM, oder zumindest ihr großer Teil, verbinden mit ihrer Unterstützung dieser Partei nicht die Vision von einer antisystemischen Politik, umso weniger den realen und durchführbaren „Umsturz", die revolutionäre Veränderung, die Rückkehr in die Zeit vor 1989 und die Wiederherstellung der "alten Ordnung" (Handl 20I0). Die Mehrheit wählt ihre Repräsentanten nicht in der sehnsüchtigen Erwartung einer radikalen Systemwende. Auch in dieser Hinsicht sind die Wähler der Partei grundsätzlich „konservativ“: Obschon sich die Wähler in der Mehrzahl die Restaurierung des alten Regimes und die Wiedereinführung seiner "Vorteile" insgeheim wünschen, haben sie sich mit dem Stand der Dinge abgefunden und ihre Identifikation mit der Partei hat alles andere als revolutionäre Wesenszüge bzw. ist der revolutionäre Inhalt dieser Identifizierung im Lauf der Zeit verblasst. Der KSČM genügt relativ wenig, um erfolgreich zu sein. Sie bedient sich einer populistischen Rhetorik vor allem in sozialen Fragen und rückt sich in die Position einer Partei, die die Interessen der unterprivilegierten sozialen Schichten vertritt (Lyons/Bernardyová 20II). Zusammenfassend können wir feststellen, dass in der KSČM sämtliche antiwestlichen, antiliberalen und antieuropäischen Ausrichtungen der tschechischen Politik bzw. der politischen Ambitionen eines Teils der Bürger Unterstützung finden. Die Agenda der KSČM nach 1989 ist systematisch durchsetzt von nationalistischer Rhetorik, und genau dieser patriotische Akzent ist im Denken der überwiegenden Mehrheit der KSČM-Wähler und -mitglieder enorm wichtig und ergänzend zu dem originären Verhältnis zur Vergangenheit ein Faktor, der die diskutierte - außerordentlich starke - „Klassenlinie“ (und die aus ihr entspringende Motivation der Wähler) überschreitet und thematisch 
erweitert und die - vom „Klassenstandpunkt“ aus eigentlich unvereinbaren - Positionen miteinander verbindet (Ishiyama 2006; Aviezer 1997).

\section{Schluss}

Es hat den Anschein, dass die tschechische Variante des Kommunismus bis heute den Bedürfnissen und Ambitionen eines bestimmten, gar nicht so kleinen Teils der tschechischen Gesellschaft Rechnung trägt. Im historischen Bewusstsein eines Teils der tschechischen Bevölkerung (und umso weniger unter den KSČM-Anhängern) entdecken wir oft nichts von der Tragik, den Verbrechen, dem zerstörten Leben (nicht nur) der Fünfzigerjahre und der Jahre der Normalisierung nach 1968, oder von unbewussten Schuldmotiven und schlechtem Gewissen. Das heißt, die „Wahrheit über den Kommunismus“ ist ebenso stark individualisiert, wie abgeleitet von der Gruppenerfahrung, und sie ist in der tschechischen öffentlichen Meinung außerordentlich vielgestaltig.

Das zentrale Paradoxon der Wende von 1989 ist: In der praktischen machtpolitischen Konstellation, aber auch auf der Ebene staatsbürgerlicher Mentalität sehen wir den Kompromiss und den kontinuierlich bestehenden Zusammenhang mit dem kommunistischen Regime (einschließlich des Fortbestehens der Partei, die es verkörperte), auf der Ebene des öffentlichen und politischen Diskurses sind wir Zeugen einer radikalen Abkehr von der jüngeren Vergangenheit und der rhetorischen Verurteilung der Verhältnisse vor 1989 und des Kommunismus im Allgemeinen. Während die Strategie der Legitimierung der Demokratie in Tschechien und der neuen politischen Eliten sich fest auf die eindeutige $\mathrm{Ab}$ lehnung der kommunistischen Vergangenheit als Ganzes stützt, lassen soziologische Untersuchungen erkennen, dass die Sicht der tschechischen Öffentlichkeit insgesamt auf die vermeintlich „abgelegte“ Vergangenheit sehr viel strukturierter ist. Eine sichtbare Bestätigung dieses „Widerspruchs in sich“ ist die legale Existenz und die überraschende Lebensfähigkeit der nicht reformierten kommunistischen Partei. Die KSČM ist die immer wieder aktualisierte Erinnerung an den besiegten Feind (Schmidt-Hartmann I992, 88).

Das Vorhandensein der kommunistischen Partei eröffnet allerdings weder eine ernstzunehmende noch eine denkbare Möglichkeit, die Diktatur stalinistischen Typs wiederherzustellen. Dennoch hat die KSČM der Gesellschaft lange Zeit als „Veranschaulichung des Bösen“ gedient und unter der unablässig wiederholten Geste der Distanzierung von ihr sind die Legitimität der neuen demokratischen Ordnung sowie bewährte Formen der politischen Meinungsbildung und der Koalitionsmathematik (re-)konstruiert worden. Nicht nur, dass sich die Kommunisten an keiner der Nachwende-Regierungen unmittelbar beteiligen durften, es galt auch für jedes Regierungskabinett als inakzeptabel, sein Bestehen auf eine kommunistische Stimme zu stützen. Dennoch gelang es der jahrelang politisch isolierten und im vorherrschenden öffentlichen Diskurs geächteten Partei, ihren Platz in der Struktur der sozialen Konfliktlinien zu finden, indem sie sich an bestimmte Teile der Bevölkerung wandte und diese politisch integrierte.

Die Wähler und Sympathisanten der KSČM sind bei Weitem keine homogene Gruppe. Es gibt unter ihnen überzeugte Verteidiger der kommunistischen Macht, Ideologie und Geschichte ebenso wie Menschen ohne konkretere ideologische Verankerung, die in der KSČM die Antwort auf bedrückende soziale Fragen suchen, und solche, die im Prinzip die demokratischen Grundwerte teilen und dem Profil der linksgerichteten Wählerschaft sozialdemokratischer oder sozialistischer Orientierung entsprechen. Die KSČM ist im Rahmen der geltenden Gesetzgebung erfolgreich tätig und sie ist fähig, mithilfe eines starken Protest-Potenzials praktisch zu allen Wahlen ihre Wähler in großer Zahl zu mobilisieren. Nach der Wende glaubten viele, der Kommunismus sei ein historisches Phänomen, das auf dem „Müllhaufen der Geschichte" gelandet sei. Es hat sich jedoch gezeigt, dass bei einem Teil der tschechischen Bevölkerung das historische Gedächtnis mit kommunistischem Gedankengut durchaus vereinbar ist. Das ermöglicht den Kommunisten, weiterhin ihren Platz im tschechischen Parteiensystem zu behaupten. Durch permanente Präsenz in diesem System ist die Partei im Lauf der Jahre zum festen Bestandteil des politischen Lebens in der Tschechischen Republik geworden. Wie die Ergebnisse der Kommunal-, Regional- und Parlamentswahlen belegen, hat die KSČM auch 26 Jahre nach dem November 1989 ihre Positionen zu halten vermocht, sie findet zeitweilig sogar neue Sympathisanten, vor allem in den sozial schwächeren bzw. gefährdeten Schichten. Und es ist offenkundig, dass die Partei ihren Platz im Parteiensystem auch weiterhin behaupten wird.

\section{Literaturverzeichnis}

Backes, Uwe/Moreau, Patrick (Hg.) (2008). Communist and Post-communist Parties in Europe, Göttingen.

Bozóki, András/Ishiyama, John T. (Hg.) (2002). The Communist Successor Parties of Central and Eastern Europe, New York.

Grzymala-Busse, Anna (1998). Reform Efforts in the Czech and Slovak Communist Parties and Their Successors I988-I993, in: East European Politics \& Societies, Vol. I2(3), 442-47I.

Grzymala-Busse, Anna (2002). Redeeming the Communist Past: The Regeneration of Communist Parties in East Central Europe, Cambridge. 
Handl, Vladimír (20I0). Living with or in the Past? Czech Communists between Canonisation and Coming to Terms with their History, in: Hofmann, Birgit (Hg.): Diktaturüberwidnung in Europa. Neue nationale und transnationale Perspektive, Heidelberg, 84-IO2.

Hanley, Seán (2002). The Communist Party of Bohemia and Moravia after 1989: Subcultural Party to Neocommunist Force?, in: Bozóki, András/Ishiyama, John T. (Hg.): The Communist Successor Parties of Central and Eastern Europe, New York, I4I-I65.

Havel, Václav (2006). Prosím stručně [Bitte kurzfassen], Prag.

Ishiyama, John T. (1999). The Communist Successor Parties and Party Organizational Development in Postcommunist Politics, in: Political Research Quaterly Vol. 52(I), 87-II2.

Ishiyama, John/Bozóki, András (200I). Adaptation and Change: Charakterizing the Survival Strategies of the Communist Successor Parties. Journal of Communist studies and Transformation Politics Vol. I7 (2), 32-5I.

Ishiyama, John (2006). Europeanization and the Communist Successor Parties in Post-Communist Politics, in: Politics \& Policy Vol. 34(I), 3-29.

Kubát, Michal (20I0). Politická opozice $\mathrm{v}$ teorii a středoevropské praxi [Politische Opposition in Theorie und mitteleuropäischer Praxis], Prag.

Kunštát, Daniel (2013). Za rudou oponou. Komunisté a jejich voliči po roce 1989 [Hinter dem roten Vorhang. Die Kommunisten und ihre Wähler seit 1989], Prag.

Mannewitz, Tom (2012). Linksextremistische Parteien in Europa nach 1990. Ursachen für Wahlerfolge und -misserfolge, Baden-Baden.

Lyons, Pat/Bernardyová, Alžběta (20II). Satisfied, Sceptical or Simply Indifferent? Current Public Opinion towards the Fall of Communism in the Czech Republic, in: Europe - Asia Studies Vol. 63(9), I719-I744.

Maškarinec, Pavel (2017). A spatial analysis of Czech parliamentary elections, 2006-2013, in: Europe-Asia Studies Vol. 69(3), 426-457.

Mateju, Petr/Kreidl, Martin (200I). Rebuilding Status Consistency in a Post-communist Society, in: Innovation: The European Journal of Social Science Research Vol. I4(I), I7-34.

Minkerberg, Michael (2000). The Renewal of the Radical Right: Between Modernity and Anti-Modernity, in: Government and Opposition Vol. 35(2): 170-188.

Moreau, Patrick (2010). Der Scheintod des Kommunismus in Europa. Ein systematischer und typologischer Überblick zu den traditionalistischen, RotGrün/linken und postkommunistischen Modellen, in: Jahrbuch für Extremismus- und Terrorismusforschung Vol. 2009/2010, 370-434.

Novotný, Lukáš (2004). Länderportrait: Tschechien, in: Jahrbuch Extremismus \& Demokratie Vol. 16, 204-220.
Novotný, Lukáš (2007). „Dann fiel der Eiserne Vorhang und das war's". Grenze in den Augen der Bürger des tschechisch-bayerischen Grenzlands, in: Neuss, Beate/Holly, Werner (Hg.): Sprache und Politik im vereinten Europa, Tübingen, I43-I66.

Novotný, Lukáš (20II). Extremismus in Tschechien, in: Jesse, Eckhard/Thieme, Tom (Hg.): Extremismus in den EU-Staaten, Wiesbaden, 397-4I2.

Oschlies, Wolf (2000). Postkommunistische Aufarbeitung in Politik und Recht der Tschechoslowakei und ihrer Nachfolgestaaten, in: Hirscher, Gerhard (Hg.): Kommunistische und postkommunistische Parteien in Osteuropa. Ausgewählte Fallstudien, München, I7-46.

Pithart, Petr (2009). Devětaosmdesátý [Das Jahr Neunundachtzig], Prag.

Putnam, Robert D. (1988). Diplomacy and Domestic Politics: The Logic of Two-Level Games in: International Organization Vol. 42(3), 427-460.

Schmidt-Hartmann, Eva (1992). Tschechoslowakei: Zwei Völker auf der Suche nach dem gemeinsamen Staat, in: Mommsen, Margarete (Hg.): Nationalismus in Osteuropa. Gefahrvolle Wege in die Demokratie, München, 77-95.

Stegmaier Mary/Vlachová Klára (20II). The Endurance of the Czech Communist Party, in: Politics \& Policy 37(4), 799-820.

Thieme, Tom (2006). Politischer Extremismus in Ostmitteleuropa - Entstehungsbedingungen und Erscheinungsformen, in: Backes, Uwe/Jesse, Eckhard (Hg.): Gefährdungen der Freiheit. Extremistische Ideologie im Vergleich, Göttingen, 32 I-358.

Thieme, Tom (2007). Hammer, Sichel, Hakenkreuz. Parteipolitischer Extremismus in Osteuropa: Entstehungsbedingungen und Erscheinungsformen, Baden-Baden.

Thieme, Tom. 2007. Durchmischung von Rechts- und Linksextremismus. Parteipolitischer Extremismus in Osteuropa, in: Jesse/Eckhard/Niedermeier, HansPeter (Hg.): Politischer Extremismus und Parteien, Berlin, 445-465.

Tucker, Aviezer/Balharova, Jana/Losman, Ivo/Němec, Jan (1997). The Czech Transition: Politics before Economics, in: The Journal of Social, Political, and Economic Studies Vol. 22(4), 395-4I6.

Vodička, Karel (2005). Das politische System Tschechiens, Wiesbaden.

Vodička, Karel (2OIO). Das politische System Tschechiens, in: Ismayr, Wolfgang (Hg.): Die politischen Systeme Osteuropas, Wiesbaden, 275-316.

Waller, Michael (1995). Adaptation of the Former Communist Parties of Eastern Central Europe. A Case of Social-democratization, in: Party Politics Vol. I(4), 473-490. 


\section{Autor}

Lukáš Novotný (Dr.) ist Politikwissenschaftler und Soziologe. Er wurde 1979 in Karlovy Vary/Karlsbad geboren und studierte in Pilsen und Bayreuth. Die Promotion erfolgte an der TU Chemnitz zum Thema der aktuellen Vergangenheitsdiskurse zwischen Deutschen und Tschechen (bei Prof. Dr. Eckhard Jesse). Heute lehrt er an der Jan-Evangelista Purkyne Universität in Ústí nad Labem sowie an der Karls-Universität Prag und beschäftigt sich schwerpunktmäßig mit den politischen Systemen der deutschsprachigen Länder. 